\title{
Is it aortic valve sclerosis a predictor for bilateral renal artery stenosis outcome in geriatric population?
}

\author{
Paloma Manea, Cristina Gavrilescu, Manuela Ursaru, \\ Corina Ursulescu, Irina Jari, Dragoş Negru, Rodica Ghiuru
}

Paloma Manea - M.D., Ph. D., Lecturer, “Grigore T. Popa” University of Medicine and Pharmacy, Iași; 1st MedicalDepartment, Vth Medical and Geriatrics - Gerontology Clinic, Iași, Senior in Internal Medicine, Specialist in Cardiology, "Promedicanon" Private Office, Iași, Romania

Cristina Gavrilescu - M.D., Ph. D., Lecturer, "Grigore T. Popa" University of Medicine and Pharmacy, Iași; 1stMedical Department, Vth Medical and Geriatrics - Gerontology Clinic, Iași; Senior in Internal Medicine, Specialist in Geriatrics, Iași, Romania

Manuela Ursaru - M.D., Ph. D, Assistant Professor,"Grigore T.Popa"University of Medicine and Pharmacy, Iasi, Surgical Department; Senior in Radiology, Iasi, Romania

Corina Ursulescu - M.D., Ph. D, Lecturer,"“Grigore T.Popa”University of Medicine and Pharmacy, Iasi, Surgical Department; Senior in Radiology, Iasi, Romania

Irina Jari - M.D., Ph. D, Lecturer,"“Grigore T.Popa”University of Medicine and Pharmacy, Iasi, Surgical Department; Senior in Radiology, Iasi, Romania

Dragoş Negru - M.D., Ph. D, Professor,“Grigore T.Popa”University of Medicine and Pharmacy, Iasi, Surgical Department; Senior in Radiology, Iasi, Romania

Rodica Ghiuru - M.D., Ph. D.,Professor, Vth Medical and Geriatrics - Gerontology Clinic, Iași; Senior in Internal Medicine, Cardiology and Geriatrics, Iasi, Romania

\footnotetext{
ABSTRACT

Introduction: It's wellknown the fact that aortic valve sclerosis (AVS) is a predictor of increased cardiovascular risk factor, but it's implication in renal artery stenosis (RAS) outcome hasn't been deepened.

Objective: The purpose was to establish a correlation between aortic valve sclerosis in geriatric patients, renal artery stenosis, vascular events and depression.

Methods: We've included 32 patients with bilateral RAS; 17 patients had also AVS (group A) and 15 patients were the control group, without AVS (group B). During 24 months, we followed the occurrence of cardiovascular events (myocardial infarction and stroke), renal failure (serum creatinine level), heart failure aggravation (echocardiographical parameters) and depression.
} 
\%ен

Results: During 24 months, the incidence of acute vascular events was higher among group A, comparatively to group B (confidence interval CI 95\%: 2.85- 2.25). Renal function was an important parameter in our study; we've observed higher serum creatinine levels for the patients with AVS, even both groups had renovascular arterial hypertension due to bilateral RAS and a physiopathological tendency to renal dysfunction (CI 95\%: 2.41 - 2.16). The heart failure prevalence was more prominent in group $A$ than group B, both systolic and diastolic types (CI 95\%: 2.76-2.3). High sensitivity C reactive protein (hs- CRP) levels were higher for group A (CI 95\%: 2.62-2.41).Different severity of depression were more frequent in group A. Conclusions: Our study revealed the fact that AVS promotes unfavorable evolution for the patients with bilateral RAS, extending its influence towards "'target" vascular territories and towards depression outcome.

\section{KEYWORDS:}

Renal artery stenosis, aortic valve sclerosis, geriatric patients, depression.

\section{INTRODUCTION}

Renovascular hypertension represents an important disorder with undesirable complications if it's not promptly diagnosed and treated (1). In 1938, Goldblatt proved that experimental hypertension can be provoked by renal ischemia (2). Since then, the researchers developed this idea and nowadays, we know that Renin- AngiotensinAldosterone System (RAAS) is a physiopathological key for hypertension and heart failure. RAS determines Renin production, which increases Angiotensin I and the last stimulates Angiotensin II. Angiotensin II is a mediator which promotes arterial hypertension through stimulating Aldosterone (increasing salt and water renal reabsorption) and through direct vasoconstriction; last decade research revealed that Angiotensin II provokes endothelial dysfunction and insulin resistance $(3,4)$. As well as renal atherosclerotic artery stenosis, aortic valve sclerosis is usually seen in elderly; AVS is a marker of increased cardiovascular events, especially myocardial infarction - 50\% prevalence in AVS patients (5). The physiopathological pathways for these two diseases- RAS and AVS- are related to sclerotic process, among heart valves and renal arteries.

\section{MATHERIALS AND METHODS}

We selected 32 patients with bilateral RAS, aged between 65 and 86 years, $71 \%$ males. The exclusion criteria were the following: diabetes mellitus, autoimmune disorders, cancer, actually smoker or alcohol drinker. The patients were diagnosed with bilateral RAS through clinical examination (periumbilical bruits), renal Doppler sonography $(6,7)$-peak velocity $\geq 1.8 \mathrm{~m} / \mathrm{s}$; resistive index $\mathrm{RI} \geq 0.7$ ( fig.1) and abdominal computed tomographic angiogram if sonography wasn't clear enough $(21 \%$ from all the patients).

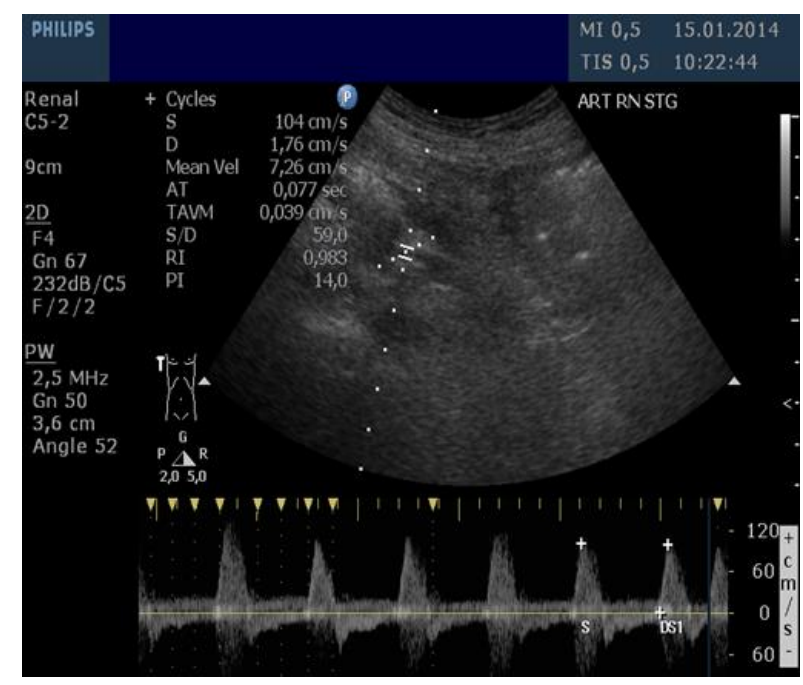

Fig.1 Resistivity index in RAS (renal artery ultrasound) (Dr. Corina Ursulescu and Dr.

Dragos Negru's collection) 
A complete examination of our patients included clinical consult, biochemical findings (serum creatinine level, transaminases, glycaemia, lipid profile, biochemical urine analysis) and complete blood count. Electrocardiogram and transthoracic echocardiography were important methods in patients' assessment. 17 patients had AVS -peak velocity $\leq 2 \mathrm{~m} / \mathrm{s}$; thickening and increased echogenity of the aortic valves, with normal leaflet movements (fig.2 and fig.3) and they were "'case group'; the other 15 patients didn't reveal this condition (AVS) at echocardiography.

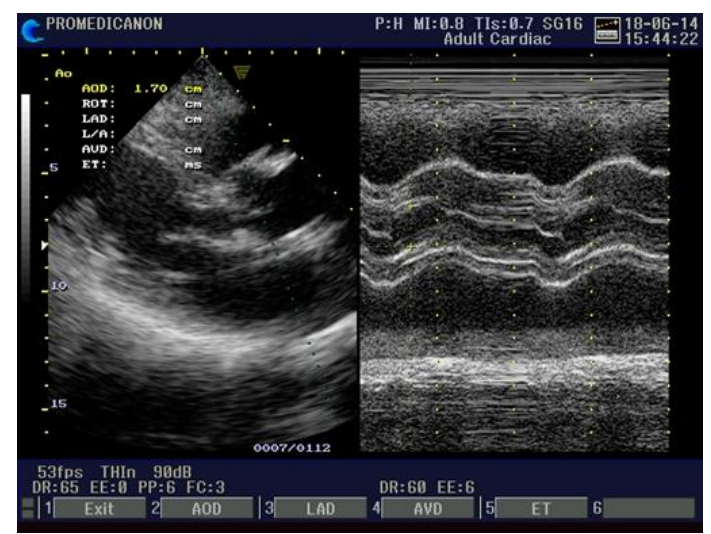

Fig.2 Aortic valve sclerosis - B and M mode echocardiography (Dr. Paloma Manea's collection)

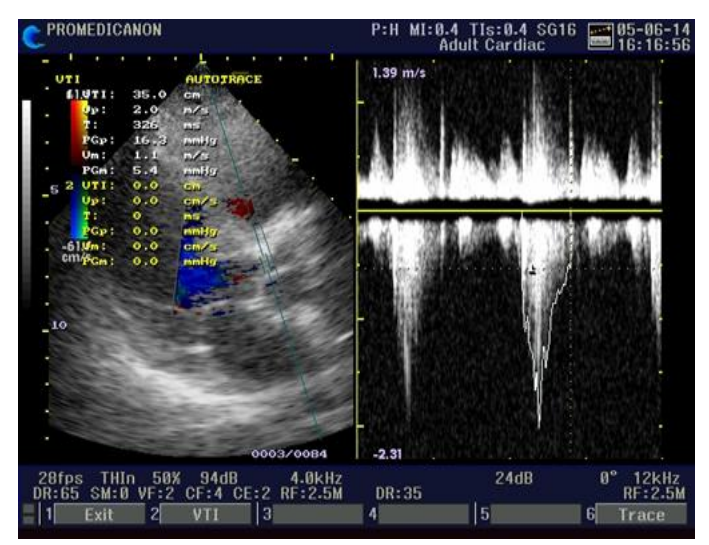

Fig.3 Transvalvular aortic gradient in AVS (Dr. Paloma Manea's collection)

Every 6 months, during 2 years, we've followed the serum creatinine level, heart failure parameters (diastolic dysfunction type; systolic dysfunction severity through echocardiography) and acute vascular events occurrence (myocardial infarction and stroke). The RAS and AVS were evaluated with the same periodicity. The patients had medical therapy for RAS, because all of them had RI $\geq$ 0.8 and this value is a good predictor of the fact that the patient doesn't benefit from revascularization (8). The antihypertensive agents were: diuretics (Indapamide 1.5 $\mathrm{mg} /$ day); calcium channel blockers Amlodipine 5-10 mg/day) and betablockers ( Nebivolol 5-10mg/day); all the patients received statins (Atorvastatin 10-40 mg/day), antiplatelet agents (Acetylsalicilic acid 100 $\mathrm{mg} /$ day or Clopidogrel $75 \mathrm{mg} /$ day) or oral anticoagulants -Acenocumarol 1-4 mg/day, if they had associated atrial fibrillation. We couldn't recommend angiotensin converting enzyme inhibitors or sartans to our patients, due to absolute contraindication of these medicines in bilateral RAS. Another parameter which raised our interest was the diagnosis of depression. For this, we evaluated the Hamilton Rating Scale for Depression, searching for insomnia, agitation, weight loss, anxiety, probing mood, suicide ideation.

\section{RESULTS}

Firstly, we followed the prevalence of acute cerebrovascular and coronary events for both groups. Even they were all treated with antihypertensive agents and we succeeded in controlling blood pressure values, at the age ad equated levels, some of our patients developed a myocardial infarction or a stroke, during these 24 months. The prevalence of these undesirable events was higher in group A (myocardial infarction $23 \%$ and stroke $17 \%$ ) than in group B (myocardial infarction $6 \%$ and stroke 6\%): CI 95\%: 2.85-2.25; $\mathrm{p}=0.008$. The patients with acute myocardial infarction or stroke received medical or interventional treatment, according to the period between the onset and admission, age 


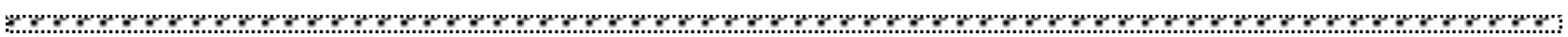

and other particularities. There was no death due to these complications. High sensitivity $\mathrm{C}$ Reactive Protein levels ( hs-CRP), as an inflammatory marker, released by adypocytes, were higher for group A than group B : CI 95\%: 2.62-2.41; $\mathrm{p}=0.009$. During last two decades, several studies noticed high levels of inflammatory markers, in patients with depression and atherosclerosis (9); these disorders had both a common backgroundreleasing of hs-CRP from adypocytes and endothelium. Secondly, we've observed the serum creatinine levels 'evolution and we've remarked that group A had higher level for this parameter than group B: CI 95\%: 2.412.16; $\mathrm{p}=0.007$. All the patients had preserved diuresis; there were 4 cases of severe worsening renal dysfunction due to urinary infection, but they promptly responded to antibiotics. Thirdly, heart failure was an important disorder which preoccupied us in this study. Group A has a higher prevalence for both diastolic and systolic function worsening (group A 18\%, comparative with group B 11\%) CI 95\%: 2.76-2.33; $\mathrm{p}=0.005$, especially for the patients with myocardial infarction. This result is explained by the reduction of systolic myocardium performance, due to ischemic heart disease and an altered relaxation. We also found a direct correlation between depression severity and the association of RAS with AVS (CI 95\%: 2.42-2.17; $\mathrm{p}=0.007)$. The most frequent symptoms related by our patients were insomnia (58\% for group A and $24 \%$ for group B) and anxiety (63\% for group A and $16 \%$ for group B).Our patients benefited by psychotherapy and antidepressants: Fluoxetine 20-40 mg/day or Mirtazapine 15-
$30 \mathrm{mg} /$ day. Hamilton scale revealed severe depression in $17 \%$ patients from group A and $6 \%$ patients from group $\mathrm{B}$; moderate depression in $23 \%$ patients from group $\mathrm{A}$ and $13 \%$ patients from group $\mathrm{B}$; mild depression was more frequent, as well as moderate and severe, between group A patients (35\%), comparative with group $\mathrm{B}(13 \%)$. These observations are concordant with other studies. Agatsa et.al noticed that patients with recurrent major depression had a higher prevalence of coronary and aortic atherosclerosis at electron beam tomography, from aortic arch to the iliac bifurcation (10). Also, in Rotterdam Study, the authors revealed a strong correlation between depression and coronary and aortic calcification (11).

\section{DISCUSSIONS}

Even in our high-specialized era, when we had to be trained in specific skills, a holistic approach of our patients brings real achievements. AVS must be seen as an "open window" to the coronary atherosclerosis. Soydine et al. established that AVS diagnosis can suggest the extent of coronary atherosclerosis (12). These authors utilized Gensini score system after coronary angiography for a correct assessment of atherosclerotic plaque burden. In our study, we noticed that AVS is both an "open window" for cerebral and renal arteries, not only for coronary territories. The endothelial dysfunction is the turntable for clinically expressed atherosclerosis and this dysfunction is strongly correlated with AVS (cardiac valves and arteries- endocardium and endothelium, similar internal layers).

\section{CONCLUSIONS}

RAS associates an increased risk of acute coronary syndrome and this risk doesn't depend on blood pressure values or other cardiovascular risk factors (13). AVS doesn't consist an "innocent" disease of the elderly; it's increasing with 50\% the risk of myocardial infarction (14). Our study concluded that AVS, associated with bilateral RAS is a negative predictor for patient's evolution, including target vascular damages: cerebral, renal and myocardial. The presence of AVS, associated with 


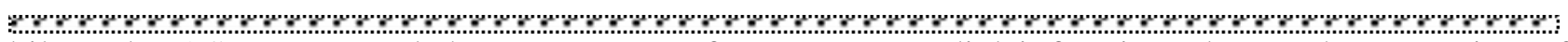
bilateral RAS, augmented the occurrence of acute myocardial infarction, due to the extension of calcium deposits from aortic valve to coronary arteries and also due to inflammatory status in AVS. The worsening of heart failure (both systolic and diastolic) for a statistically significant number of patients with AVS is correlated with the evolution of ischemic heart disease in AVS. Serum creatinine levels were influenced by AVS association, due to continuum cardio-renal relationship (endocardium status in AVS and renal artery endothelium in bilateral RAS. Patients with depression, from our study, revealed a direct correlation between depression severity and AVS, due to major turntable hs-CRP, significantly increased in both these diseases, cardiological and psychiatric ones. Future studies will include a larger number of patients and we have to extend inflammatory markers to the assessment of tumor necrosis factor receptors and interleukin 6 .

\section{ACKNOWLEDGE AND DISCLOSURE}

The authors declare they have no potential conflicts of interest to disclose.

\section{REFERENCES}

1. Edwuards MS, Craven TE, Burke GL, Dean RH, Hansen KJ. Renovascular disease and the risk of adverse coronary events in the elderly: a prospective, population- based study. Arch Intern Med. 2005; 165 (2): 207-213

2. Goldblatt H. Experimental hypertension induced by renal ischemia, Harvey Lecture, May 19,1938. Bull N Y Acad Med. 1938; 14 (9) : 523- 553

3. Khosla S,Ahmed A, Siddiqui M et al.Safety of angiotensin-converting enzyme inhibitors in the bilateral artery stenosis following succesfull renal artery stent revascularization.Am J Ther.2006;13(4):306-308

4. Nakashima H, Suzuki H, Ohtsu H et al. Angiotensin II regulates vascular and endothelial dysfunction : recent topics of Angiotensin II type - 1 receptor signaling in the vasculature. Curr Vasc Pharmacol. 2006; 4 (1): 67-68

5. Nightingale AK, Horowitz JD. Aortic sclerosis: not an innocent murmur but a marker of increased cardiovascular risk.Heart.2005;91(11): 1389-1393

6. Rademacher J, Ellis S .Haller H. Renal resistance index and progression of renal disease. Hypertension.2002; 39(2 Pt 2): 699-703

7. Tublin ME, Bude RO, Platt JF. The resistive index in renal Doppler sonography:where do we stand? Am J Roentgenol.2003;180: 885-892

8. Bruno RM, Daghini E, Versari D et al. Predictive role of renal resistive index for clinical outcome after revascularization in hypertensive patients with atherosclerotic renal artery stenosis: a monocentric observational study.Cardiovasc Ultrasound. 2014; 18:9

9. Raikkonen K, Mathews KA, Kuller LH et al. The relationship between psychological risk attributes and the metabolic syndrome in healthy women: antecedent or consequence? Metabolism. 2002; 51:1537-1577

10. Agatsa P, Mathwes KA, Bromberger JT et al. Coronary and aortic calcificationin women with a history of major depression.Arch Intern Med. 2005;165(11): 1229-1236

11. Tienmeier H, Hoffman WA, Wittterman JCM et al.Relantionship between atherosclerosis and late-life depressionthe Rotterdam Study. Arch Ger Psychiatry. 2004; 61:369-376

12. Soydine S, Danritoglu V, Dundar A et al. Relationship between aortic valve sclerosis and the extent of coronary artery disease in patients undergoing diagnostic coronary angiography. Cardiology. 2006; 106(4): 277-282

13. Payami B, Jafarizade M, Mousari SSB et al. Prevalence and predictors of atherosclerotic renal artery stenosis in hypertensive patients undergoing simultaneous coronary and renal artery angiography; a cross-sectional study. J Renal Inj Prev. 2016; 5(1): 34-38.

\section{Correspondence:}

Paloma Manea,

Șos. Bucium no.6, 700282, Iasi, Romania, +40722569770, maneacpaloma@yahoo.com 